\title{
Recuperação de Portadora Tipo Feedforward para Sistemas 8-PSK com Multiplexação de Polarização
}

\author{
Francisco A. C. Garcia, Darli A. A. Mello, Helio Waldman
}

\begin{abstract}
Resumo-Comparamos o desempenho de quatro arquiteturas de recuperação de portadora tipo feedforward em receptores 8-PSK coerentes com multiplexação de polarização. Analisamos os cenários que apresentam desequilíbrio entre as SNRs nas duas polarizações, tal como ocorre em sistemas com PDL. Usando simulações de Monte Carlo, observamos que o método de estimação conjunta proposto em [1] apresenta penalidade em SNR de 1,1 dB para uma razão largura de linha/taxa de símbolo $=7,5 \times 10^{-4}$, enquanto as outras três arquiteturas investigadas têm penalidade $1,5 \mathbf{d B}, 1,7$ dB e $2,8 \mathrm{~dB}$, para BER $=10^{-3}$ e um desequilíbrio em SNR de 3 dB.
\end{abstract}

Palavras-Chave-Comunicações ópticas, detecção coerente, modulação de fase.

Abstract-We investigate four feedforward carrier recovery (FFCR) architectures for coherent polarization multiplexing 8PSK receivers where the signal to noise ratios (SNR) of polarization demultiplexed signals can be unequal, such as in PDL impaired systems. We evaluated each architecture using Monte Carlo simulations and observed that the joint-polarization FFCR proposed in [1] yields a $1.1 \mathrm{~dB}$ SNR penalty for a given laser linewidth/baud rate $=7.5 \times 10^{-4}$ ratio, while the other three architectures offer $1.5 \mathrm{~dB}, 1.7 \mathrm{~dB}$ and $2.8 \mathrm{~dB}$, at BER $=10^{-3}$ and $3 \mathrm{~dB}$ SNR imbalance. The joint-polarization architecture is therefore an attractive alternative for 8-PSK modulated systems.

Keywords-Optical communications, coherent detection, phase modulation.

\section{INTRODUÇÃO}

A detecção coerente de sinais ópticos em sistemas com multiplexação por divisão por comprimento de onda (WDM) torna-se imprescindível à medida que aumentam a taxas de transmissão por canal. Há três motivos essenciais: o aumento da sensibilidade do receptor, a compensação eficiente de efeitos lineares intrínsecos à transmissão pela fibra óptica, e a separação linear de sinais multiplexados em polarizações ortogonais. Um componente importante do detector coerente é a recuperação de portadora, responsável por compensar a diferença de fase resultante de processos ruidosos nos lasers transmissor e oscilador local. Nosso grupo de pesquisa propôs recentemente um mecanismo de recuperação de portadora tipo feedforward (FFCR) que utiliza conjuntamente informações sobre o ruído de fase nas duas polarizações ortogonais. O mecanismo é especialmente interessante em sistemas nos quais

Francisco A. C. Garcia. Departamento de Comunicações, Faculdade de Engenharia Elétrica e Computação, Universidade Estadual de Campinas, SP, Brasil, E-mail: fgarcia@decom.fee.unicamp.br. Darli A. A. Mello. Departamento de Engenharia Elétrica, Faculdade de Tecnologia, Universidade de Brasília, DF, Brasil, E-mail: darli.mello@ene.unb.br. Helio Waldman. OptiNet, UFABC, Santo André, SP, Brasil, E-mail: helio.waldman@ufabc.edu.br. Este trabalho foi parcialmente financiado pela FAPESP. a relação sinal-ruído (SNR) nas duas polarizações é desigual (assumimos a separação perfeita das duas polarizações) [1], como pode acontecer em sistemas sujeitos à perda dependente da polarização (PDL) [2]. Além de propor o novo método, avaliamos em [1] seu desempenho em sinais com modulação QPSK. Neste trabalho, alternativamente, comparamos o desempenho do método proposto em [1] ao de três outros métodos quando aplicados em sinais com modulação 8-PSK, para a qual a influência do ruído de fase é mais severa.

\section{Modelo DO SISTEMA}

No transmissor, dois sinais complexos modulam o laser transmissor por meio de dois moduladores Mach-Zehnder I+Q (I+Q MZM) em fase e em quadratura, a fim de produzir dois sinais 8-PSK. Esses sinais são então acoplados em um combinador de polarização (polarization beam combiner PBC). O resultado é um sinal multiplexado em polarização (polarization multiplexed signal - PolMux). A representação complexa do sinal PolMux, $\mathbf{s}_{k}$, em banda base é dada por [1]:

$$
\mathbf{s}_{k}=\left[\begin{array}{c}
s_{k}^{V} \\
s_{k}^{H}
\end{array}\right],
$$

em que $s_{k}^{V}$ e $s_{k}^{H}$ são os símbolos transmitidos nas polarizações vertical $(V)$ e horizontal $(H)$.

Em seu percurso pela fibra (Fig. 1), o sinal PolMux é corrompido pela PDL e pelo ruído aditivo de emissão espontânea amplificada (amplified spontaneous emission - ASE) advindo dos estágios de amplificação (EDFA). No receptor, o sinal é misturado ao oscilador local (local oscillator - LO) por um conjunto de híbridas de $90^{\circ}$. O sinal é então detectado nos fotodetectores balanceados, seguindo para um banco de filtros casados aos sinais transmitidos. Assume-se amostragem perfeita.

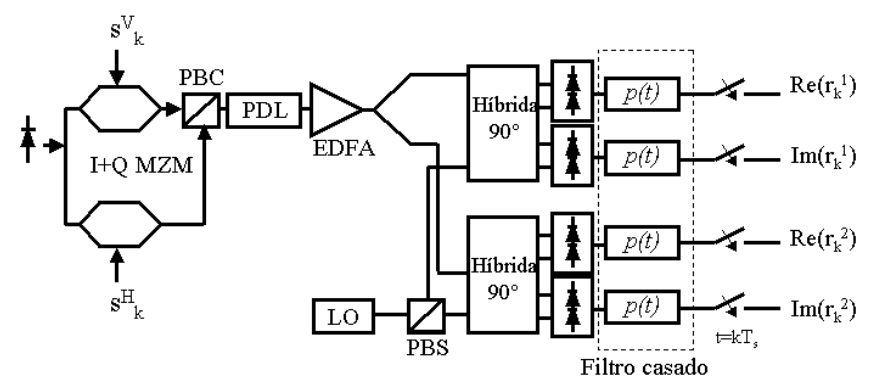

Fig. 1. Sistema de multiplexação de polarização em um receptor homódino.

Após a devida equalização e separação de polarização, os sinais podem ser representados por:

$$
\mathbf{y}_{k}=\mathbf{s}_{k} \exp \left(j \theta_{k}\right)+\mathbf{w}_{k}
$$


em que $\mathbf{y}_{k}$ é um vetor com os sinais nas duas polarizações, $\mathbf{s}_{k}$ é o vetor dos sinais transmitidos, $\theta_{k}$ o ruído de fase (escalar), e $\mathbf{w}_{k}$ um vetor de ruídos aditivos. Embora os ruídos aditivos sejam processos independentes em cada polarização, o ruído de fase, por sua vez, é o mesmo. Pode-se representar a equação acima de forma escalar:

$$
y_{k}^{V / H}=s_{k}^{V / H} \exp \left(j \theta_{k}\right)+w_{k}^{V / H},
$$

em que $\sigma_{w}^{2}{ }^{H}$ pode ser diferente de $\sigma_{w}^{2}{ }^{V}$ (por exemplo, na presença de PDL).

\section{RECUPERAÇÃO DE PORTADORA TIPO FEEDFORWARD}

\section{A. Uma polarização}

Mecanismos de recuperação de portadora estimam o ruído de fase $\theta_{k}$ a partir de sinais $y_{k}$ corrompidos por ruído aditivo e ruído de fase (usualmente, um processo de Wiener):

$$
y_{k}=s_{k} \exp \left(j \theta_{k}\right)+w_{k},
$$

em que $w_{k}$ é um processo aleatório AWGN com média nula e variância $\sigma_{w}^{2}=N_{0}$. Um mecanismo clássico de recuperação de portadora é o de Viterbi e Viterbi [3] (veja a Fig. 2). $\mathrm{O}$ primeiro passo do algoritmo é isolar as contribuições de ruído e informação do sinal recebido. Em sistemas $M$-PSK, isso pode ser obtido elevando o sinal complexo à $M$-ésima potência. Depois disso, filtram-se os ruídos correspondentes a cada amostra para mitigar a influência do ruído aditivo. Em seguida, toma-se o argumento de cada amostra e o divide por $M$. Segue-se então um dispositivo de phase unwrapping (PU), para permitir que a fase estimada $\hat{\theta}_{k}$ possa excursionar de $-\infty$ $\mathrm{a}+\infty$, em vez de $-\pi / M$ a $+\pi / M[4]$ :

$$
P U(\cdot)=(\cdot)+\left(\left\lfloor\frac{1}{2}+\frac{\hat{\theta}_{k-1}-(\cdot)}{2 \pi / M}\right\rfloor\right) \frac{2 \pi}{M} .
$$

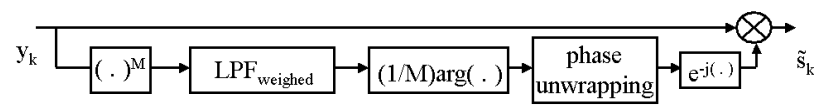

Fig. 2. Recuperação de portadora do tipo feedforward para um sistema com única polarização.

O ruído de fase $\theta_{k}$ é um processo discreto de Wiener:

$$
\begin{array}{r}
\theta_{k}=\theta_{k-1}+\Delta_{k} ; \\
\theta_{k-i}=\theta_{k}+\sum_{m=0}^{i-1} \lambda_{m} ; \\
\theta_{k+i}=\theta_{k}+\sum_{m=0}^{i-1} \mu_{m},
\end{array}
$$

em que $\Delta_{k}, \lambda_{m}$ e $\mu_{m}$ são variáveis aleatórias com distribuição gaussiana de média nula e variância $\sigma_{\Delta}^{2}=\sigma_{\lambda}^{2}=\sigma_{\mu}^{2}=$ $2 \pi \Delta \nu T_{s}$. A largura de linha $\Delta \nu$ é a soma das bandas a $3 \mathrm{~dB}$ do laser transmissor e do oscilador local (LO). O período de símbolo é $T_{s}$. As amostras passadas elevadas à $M$-ésima potência podem ser escritas como [5], [1]:

$$
\begin{aligned}
y_{k-i}^{M} & =\left\{s_{k-i} \exp \left[j\left(\theta_{k}+\sum_{m=0}^{i-1} \lambda_{m}\right)\right]+w_{k-i}\right\}^{M} \\
& \approx s_{k-i}^{M} \exp \left[j M\left(\theta_{k}+\sum_{m=0}^{i-1} \lambda_{m}\right)\right]+z_{k-i},
\end{aligned}
$$

em que $z_{k-i}$ é uma variável aleatória gaussiana com média nula e variância $\sigma_{z}^{2}=M^{2} E_{s}^{M-1} \sigma_{w}^{2}$, e com $E_{s}=\left|s_{k}\right|^{2}$. Assumindo o ruído de fase suficientemente pequeno:

$$
y_{k-i}^{M} \approx E_{s}^{M / 2} \exp \left(j M \theta_{k}\right)\left(1+j M \sum_{m=0}^{i-1} \lambda_{m}\right)+z_{k-i}
$$

De forma análoga, os símbolos futuros podem ser escritos como:

$$
y_{k+i}^{M} \approx E_{s}^{M / 2} \exp \left(j M \theta_{k}\right)\left(1+j M \sum_{m=0}^{i-1} \mu_{m}\right)+z_{k+i} .
$$

Observando a função densidade de probabilidade conjunta do vetor recebido $\mathbf{r}=\left[y_{k-N}^{M}, \ldots, y_{k-1}^{M}, y_{k}^{M}, y_{k+1}^{M}, \ldots, y_{k+N}^{M}\right]^{T}$, e maximizando-a a fim de encontrar uma estimativa $\tilde{\theta}_{k}$, temse:

$$
\tilde{\theta}_{k}=\frac{1}{M} \arctan \frac{\Im\left\{\mathbf{1}^{T} \mathbf{C}^{-1} \cdot \mathbf{r}\right\}}{\Re\left\{\mathbf{1}^{T} \mathbf{C}^{-1} \cdot \mathbf{r}\right\}} .
$$

A matriz C é a matriz de correlação dada por:

$$
\mathbf{C}=E_{s}^{M} M^{2} \mathbf{K} \sigma_{\Delta}^{2}+E_{s}^{M-1} M^{2} \mathbf{K}_{n}
$$

em que $\mathbf{K}_{n}=\sigma_{w}^{2} \mathbf{I}_{L \times L}$ para um filtro de comprimento $L=$ $2 N+1$, e $\mathbf{K}$ é dado por:

$$
\mathbf{K}=\left[\begin{array}{ccccccccc}
N & \cdots & 2 & 1 & 0 & 0 & 0 & \cdots & 0 \\
\vdots & \ddots & \vdots & \vdots & \vdots & \vdots & \vdots & . & \vdots \\
2 & \cdots & 2 & 1 & 0 & 0 & 0 & \cdots & 0 \\
1 & \cdots & 1 & 1 & 0 & 0 & 0 & \cdots & 0 \\
0 & \cdots & 0 & 0 & 0 & 0 & 0 & \cdots & 0 \\
0 & \cdots & 0 & 0 & 0 & 1 & 1 & \cdots & 1 \\
0 & \cdots & 0 & 0 & 0 & 1 & 2 & \cdots & 2 \\
\vdots & . & \vdots & \vdots & \vdots & \vdots & \vdots & \ddots & \vdots \\
0 & \cdots & 0 & 0 & 0 & 1 & 2 & \cdots & N
\end{array}\right]
$$

Levando em conta o diagrama da Fig. 2, a estimativa de $\theta_{k}$ após o PU, $\hat{\theta}_{k}$, é representada por:

$$
\hat{\theta}_{k}=P U\left\{\frac{1}{M} \arg \left(\mathbf{1}^{T} \mathbf{C}^{-1} \cdot \mathbf{r}\right)\right\}
$$

\section{B. Duas polarizações}

Apresentamos agora o caso no qual existem duas polarizações. Investigamos quatro arquiteturas de recuperação de portadora que se diferem essencialmente nos pesos do filtro passa-baixas e na localização do PU. 
1) Estimação individual: Nessa arquitetura, utilizam-se as amostras em cada polarização individualmente (veja Fig. 3). As estimativas $\hat{\theta}_{k}^{V}$ e $\hat{\theta}_{k}^{H}$ resultam da filtragem dos símbolos recebidos elevados à $M$-ésima potência:

$$
\hat{\theta}_{k}^{V / H}=P U\left\{\frac{1}{M} \arg \left[\mathbf{1}^{T}\left(\mathbf{C}^{V / H}\right)^{-1} \cdot \mathbf{r}^{V / H}\right]\right\} .
$$

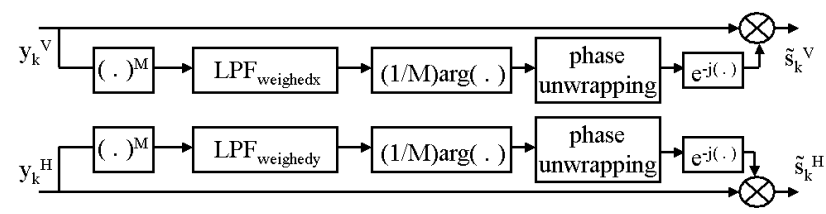

Fig. 3. Estimação individual do ruído de fase para um sistema com diversidade de polarização.

2) Estimação média: Usando dos mesmos filtros da estimação individual, calcula-se a média aritmética dos fasores complexos, gerando uma única estimativa $\hat{\theta}_{k}$ (veja a Fig. 4):

$$
\hat{\theta}_{k}=P U\left\{\frac{1}{M} \arg \left[\frac{\mathbf{1}^{T} \mathbf{C}^{V^{-1}} \cdot \mathbf{r}^{V}+\mathbf{1}^{T} \mathbf{C}^{H^{-1}} \cdot \mathbf{r}^{H}}{2}\right]\right\} .
$$

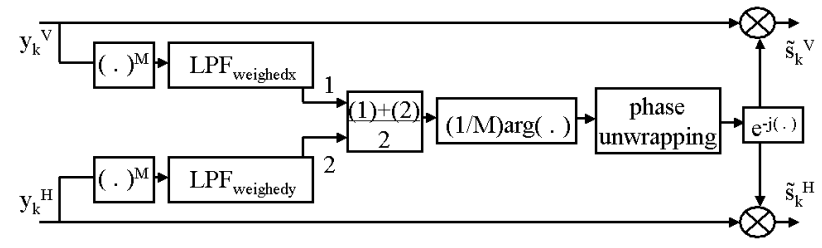

Fig. 4. Recuperação de portadora utilizando a média das estimativas individuais nas duas polarizações.

3) Estimação com filtro plano: Por ser de relativa facilidade de implementação, a arquitetura de estimação de $\theta_{k}$ por meio de um filtro plano finito vem sendo largamente utilizada. $\mathrm{O}$ tamanho usado para o filtro é escolhido de forma a minimizar a taxa de erro de bits (bit error rate - BER) (tal informação pode ser recebida, por exemplo, pelo mecanismo de correção de erro). Uma expressão analítica para o tamanho ótimo do filtro pode ser encontrado em [6].

$$
\hat{\theta}_{k}=P U\left\{\frac{1}{M} \arg \left(\frac{\sum_{i=-N}^{N} y_{k+i}^{V}{ }^{M}+\sum_{i=-N}^{N} y_{k+i}^{H}{ }^{M}}{2}\right)\right\},
$$

4) Estimação conjunta: Nosso grupo propôs em [1] um método de estimação conjunta do ruído de fase a partir das amostras em ambas as polarizações. Para tanto, deve-se

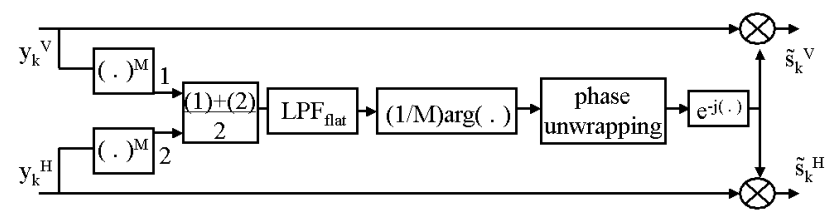

Fig. 5. Recuperação de portadora com um filtro plano em duas polarizações.

maximizar a função $f_{\mathbf{r}^{\prime} \mid \theta_{k}}\left(\mathbf{r}^{\prime} \mid \theta_{k}\right)$, tendo como vetor recebido:

$$
\begin{aligned}
\mathbf{r}^{\prime}= & {\left[y_{k-N}^{V}{ }^{M}, \ldots, y_{k}^{V^{M}}, \ldots,\right.} \\
& \left.y_{k+N}^{V}{ }^{M}, y_{k-N}^{H}{ }^{M}, \ldots, y_{k}^{H^{M}}, \ldots, y_{k+N}^{H}{ }^{M}\right]^{T} .
\end{aligned}
$$

Note que r' inclui amostras das polarizações vertical e horizontal. A matriz $\mathbf{C}^{\prime}$ associada ao vetor $\mathbf{r}$ ' pode ser descrita como:

$$
\mathbf{C}^{\prime}=E_{s}^{M} M^{2} \mathbf{K}^{\prime} \sigma_{\Delta}^{2}+E_{s}^{M-1} M^{2} \mathbf{K}^{\prime}{ }_{n},
$$

em que $\mathbf{K}^{\prime}$ e $\mathbf{K}^{\prime}{ }_{n}$ são obtidas a partir da matriz $\mathbf{K}$ definida na Seção III-A:

$$
\begin{gathered}
\mathbf{K}^{\prime}=\left[\begin{array}{cc}
\mathbf{K} & \mathbf{K} \\
\mathbf{K} & \mathbf{K}
\end{array}\right] \\
\mathbf{K}^{\prime}{ }_{n}=\left[\begin{array}{cc}
\mathbf{I}_{L \times L} \sigma_{w_{n}^{V}}^{2} & \mathbf{0} \\
\mathbf{0} & \mathbf{I}_{L \times L} \sigma_{w_{n}^{H}}^{2}
\end{array}\right] .
\end{gathered}
$$

Finalmente, o ruído de fase é estimado por:

$$
\hat{\theta}_{k}=P U\left\{\frac{1}{M} \arg \left[\mathbf{1}^{T} \mathbf{C}^{,-1} \cdot \mathbf{r},\right]\right\} .
$$

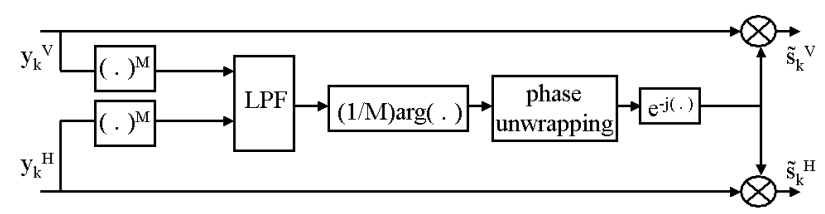

Fig. 6. Recuperação de portadora por meio de estimação conjunta das amostras nas duas polarizações.

\section{AVAliaçÃo DE DESEMPEnHo}

Avaliamos o desempenho dos quatro métodos de recuperação de portadora usando simulações de Monte Carlo. No transmissor, geramos dois sinais 8-PSK com codificação de Gray (polarizações vertical $V$ e horizontal $H$ ), produzidos a partir de quatro seqüências pseudo-aleatórias. Para incorporar o ruído de fase, multiplicamos os sinais nas duas polarizações igualmente por um processo discreto de Wiener com $\sigma_{\Delta}^{2}=2 \pi \Delta \nu T_{s}$. Assumindo separação de polarização perfeita, adicionamos ruído AWGN com $3 \mathrm{~dB}$ de desequilíbrio às duas polarizaçoes $\left(\sigma_{w}^{2} H=2 \sigma_{w}^{2} V\right)$.

$\mathrm{O}$ receptor consiste de um dos quatro métodos de recuperação de portadora, seguido de um estimador de distância mínima e posterior decodificação diferencial. A taxa 
de erro de bit (BER) para cada polarização é calculada para pelo menos $3 \times 10^{6}$ bits. Na Fig. 7 , as penalidades foram obtidas pela diferença (em $\mathrm{BER}=10^{-3}$ ) entre os valores experimentais (oferecidos pela simulação) e valores teóricos de SNR, calculados pelo inverso da Eq. 23 [5]:

$$
\operatorname{BER}\left(S N R=\frac{E_{b}}{N_{0}}\right) \approx \frac{2 Q}{\log _{2} M}\left[\sin \left(\frac{\pi}{M}\right) \sqrt{\frac{2 E_{b} \log _{2} M}{N_{0}}}\right] .
$$

Utilizamos um passo máximo de $0,1 \mathrm{~dB}$ de SNR, e o tamanho do filtro na recuperação de portadora foi de $L=$ $2 N+1=21$. Para o caso de estimação por filtro plano, o tamanho do filtro é tal que minimize a taxa de erro de bit (BER), até um tamanho máximo de $L=2 N+1=21$. Dessa forma, variamos $N$ de 1 a 10 e escolhemos o tamanho do filtro que resulta na menor BER. A Fig. 7 refere-se à polarização mais degradada. Afora um aumento geral da susceptibilidade ao ruído de fase, os resultados indicam um comportamento semelhante àquele observado com modulação QPSK, investigada em [1]. A arquitetura FFCR com estimação conjunta apresenta uma penalidade de $1,1 \mathrm{~dB}$ na SNR para uma dada razão largura de linha/taxa de símbolo de $7,5 \times 10^{-4}$, enquanto as outras arquiteturas têm penalidade de 1,5 dB (média), 1,7 dB (filtro plano) e 2,8 dB (individual).

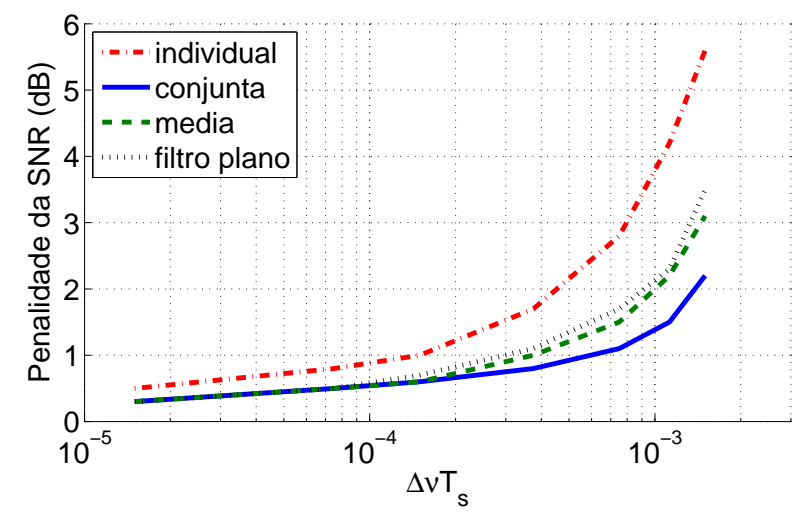

Fig. 7. Desempenho das quatro arquiteturas FFCR na pior polarização medida pela penalidade da SNR versus a razão largura de linha/taxa de símbolo para uma modulação 8-PSK com desequilíbrio de $3 \mathrm{~dB}$ de SNR entre as polarizações e BER $=10^{-3}$.

\section{CONCLUSÃO}

Investigamos o desempenho do mecanismo de recuperação de portadora proposto em [1] em sistemas com modulação 8-PSK. Anteriormente, o mecanismo havia sido testado em sistemas QPSK com sucesso. Simulações de Monte Carlo indicam, como esperado, que os sistemas 8-PSK são mais susceptíveis ao ruído de fase que sistemas QPSK, e que o mecanismo de recuperação de portadora proposto em [1] continua sendo mais eficiente que as outras três técnicas estudadas neste artigo. Quantitativamente, a arquitetura FFCR com estimação conjunta apresenta uma penalidade de $1,1 \mathrm{~dB}$ na SNR para uma dada razão largura de linha/taxa de símbolo $=7,5 \times$ $10^{-4}$, enquanto as outras arquiteturas têm penalidade de 1,5 $\mathrm{dB}$ (média), 1,7 dB (filtro plano) e 2,8 dB (individual).

\section{REFERÊNCIAS}

[1] F. A. C. Garcia, D. A. A. Mello, H. Waldman, "Feedforward Carrier Recovery for Polarization Demultiplexed Signals with Unequal Signal to Noise Ratios," Opt. Express 17, 7958-7969 (2009), http://www.opticsinfobase.org/oe/abstract.cfm?uri=oe-17-10-7958.

[2] T. Duthel, C.R.S. Fludger, J. Geyer and C. Schulien, "Impact of polarisation dependent loss on coherent POLMUX-NRZ-DQPSK," Optical Fiber Communications conference, Paper OThU5, OFC San Diego (2008).

[3] A. J. Viterbi and A. M. Viterbi, "Nonlinear Estimation of PSKModulated Carrier Phase with Application to Burst Digital Transmission,” IEEE Trans. Inf. Theory 29, 543-551 (1983).

[4] E. Ip and J. M. Kahn, "Digital equalization of chromatic dispersion and polarization mode dispersion," J. Lightwave Technol. 25, 2033-2043 (2007).

[5] E. Alpman, "Estimation of oscillator phase noise for MPSK-based communication systems over AWGN channels," theses for degree of master of science (2004), http://citeseerx.ist.psu.edu/viewdoc/summary?doi=10.1.1.2.8940.

[6] G. Goldfarb and G. Li, "BER estimation of QPSK homodyne detection with carrier phase estimation using digital signal processing," Opt. Express 14, 8043-8053 (2006), http://www.opticsinfobase.org/abstract.cfm?URI=oe-14-18-8043. 